\title{
O farmacêutico clínico na farmácia comunitária privada: revisão integrativa
}

\author{
The clinical pharmacist in the private community pharmacy: integrative review
}

El farmacéutico clínico en la farmácia comunitaria privada: revisión integradora

Recebido: 07/10/2021 | Revisado: 12/10/2021 | Aceito: 19/10/2021 | Publicado: 21/10/2021

\author{
Daniel Santana dos Santos \\ ORCID: https://orcid.org/0000-0001-5799-4497 \\ Faculdade Integrada Carajás, Brasil \\ E-mail: danielbiofarma@gmail.com \\ Yolanda de Jesus Morais \\ ORCID: https://orcid.org/0000-0001-7105-8267 \\ Faculdade Integrada Carajás, Brasil \\ E-mail: yolandamorais123@gmail.com
}

\begin{abstract}
Resumo
A implantação dos serviços clínicos farmacêuticos foi iniciada em ambiente hospitalar e posteriormente expandida para farmácias comunitárias privadas. o objetivo do artigo é a realização de uma síntese de evidências sobre a importância do farmacêutico clínico na farmácia comunitária privada proporcionando aos utentes tratamentos eficientes e com a melhoria da qualidade de vida. Método: Trata-se de uma revisão integrativa fundamentada na pesquisa bibliográfica, método que agrupa a produção científica relevante acerca de um tema pré estabelecido. Resultados e discussão: 14 artigos foram selecionados, sendo 08 deles revisões de literatura, 01 estudo transversal, 01 revisão sistemática com síntese narrativa e 04 estudos bibliográficos integrativos, sendo um dele com estudo quantitativo. Os estudos contemplem e sintetizam a importância do farmacêutico clínico na farmácia comunitária privada. Conclusão: As evidências apresentadas neste estudo demonstram a ação do farmacêutico clinico na farmácia comunitária, demonstrando que cabe a este profissional o manejo, dispensação e orientação quanto ao uso de medicamentos.
\end{abstract}

Palavras-chave: Farmácia clínica; Farmacêutico; Serviços clínicos.

\begin{abstract}
The implementation of clinical pharmaceutical services was initiated in a hospital environment and later expanded to private community pharmacies. The aim of the article is to carry out a synthesis of evidence on the importance of the clinical pharmacist in the private community pharmacy, providing users with efficient treatments and improving the quality of life. Method: This is an integrative review based on bibliographical research, a method that groups the relevant scientific production on a pre-established topic. Results and discussion: 14 articles were selected, being 08 of them literature reviews, 01 cross-sectional study, 01 systematic review with narrative synthesis and 04 integrative bibliographic studies, one of which was a quantitative study. The studies contemplate and synthesize the importance of the clinical pharmacist in private community pharmacy. Conclusion: The evidence presented in this study demonstrates the action of the clinical pharmacist in community pharmacy, demonstrating that this professional is responsible for handling, dispensing and providing guidance on the use of medications.
\end{abstract}

Keywords: Clinical pharmacy; Pharmaceutical; Clinical services.

\section{Resumen}

La implementación de los servicios farmacéuticos clínicos se inició en un entorno hospitalario y luego se expandió a las farmacias comunitarias privadas. El objetivo del artículo es realizar una síntesis de evidencias sobre la importancia del farmacéutico clínico en la farmacia comunitaria privada, proporcionando a los usuarios tratamientos eficientes y mejorando la calidad de vida. Método: Se trata de una revisión integradora basada en la investigación bibliográfica, método que agrupa la producción científica relevante sobre un tema preestablecido. Resultados y discusión: Se seleccionaron 14 artículos, siendo 08 de ellos revisiones de literatura, 01 estudio transversal, 01 revisión sistemática con síntesis narrativa y 04 estudios bibliográficos integradores, uno de los cuales fue un estudio cuantitativo. Los estudios contemplan y sintetizan la importancia del farmacéutico clínico en la farmacia comunitaria privada. Conclusión: La evidencia presentada en este estudio demuestra la acción del farmacéutico clínico en la farmacia comunitaria, demostrando que este profesional es el responsable del manejo, dispensación y orientación sobre el uso de medicamentos.

Palabras clave: Farmacia clínica; Farmacéutico; Servicios clínicos. 


\section{Introdução}

A implantação dos serviços clínicos farmacêuticos foi iniciada em ambiente hospitalar e posteriormente expandida para farmácias comunitárias privadas. No intuito de promover a saúde coletiva e individual de cada paciente. Nos últimos anos, as mudanças nas legislações propiciaram a implantação de consultórios farmacêuticos autônomos, dando uma visibilidade maior para os serviços clínicos farmacêuticos (Amariles et al., 2020).

As condutas do farmacêutico clínico buscam otimizar a farmacoterapia, promover o uso racional de medicamentos e, sempre que possível, melhorar a qualidade de vida do paciente. O Farmacêutico clínico na farmácia comunitária privada tem inúmeras atribuições, como: analisar prescrições, desenvolver ações para promoção, proteção, recuperação da saúde e a prevenção de doenças aos seus utentes, realizar a gestão de toda a farmácia (Brasil, 2018).

Os serviços clínicos executados pelo farmacêutico, favorece a adesão a terapia medicamentosa, minimizam os erros relacionados a administração dos medicamentos e principalmente proporciona qualidade de vida para os utentes, em especial os que apresentam alguma patologia crônica e estão descompensados devido ao uso inadequado dos medicamentos ou até mesmo ausência do uso (Da Cruz, Queiroz \& Soler, 2020).

O cuidado farmacêutico é centrado no utente, nele o profissional elabora um plano de cuidado de acordo com os sinais e sintomas apresentados pelo utente. É importante ressaltar que esse plano de cuidado necessita ser realizado em comum acordo com o utente, favorecendo o elo de confiança entre o utente e o profissional farmacêutico (Da Cruz, Queiroz \& Soler, 2020).

A elaboração desse artigo justifica-se devido a necessidade de descrever a importância do farmacêutico clínico na farmácia comunitária privada e os benefícios oriundos da atuação desse profissional, que garante o direito de informação corretaao utente, destacando o verdadeiro papel do farmacêutico como um profissional de saúde.

Nesse contexto de ascensão da profissão farmacêutica, com uma maior visibilidade dos serviços clínicos prestados pelo farmacêutico por toda a comunidade, o objetivo do artigo é a realização de uma síntese de evidências sobre a importância do farmacêutico clínico na farmácia comunitária privada proporcionando aos utentes tratamentos eficientes e com a melhoria da qualidade de vida.

\section{Evolução da farmácia comunitária no Brasil}

A farmácia comunitária brasileira tem sua característica estabelecida na evolução de avanços acentuada ao atendimento explicito na adequação da Orientação farmacêutica, contendo a inovação na prestação do serviço farmacêutico, considerando-se estabelecimentos com a importância de recursos adequados para cuidados com saúde.

No Brasil, as empresas do segmento farmacêutico destacando-se a farmácia comunitária privada e o ramo de medicamentos, continuam crescendo e evoluindo acima do Produto Interno Bruto (PIB), sendo assim um ramo bastante atrativo, se destacando como empresas que buscam entender a necessidade do mercado, oferecendo produtos e serviços, atenção farmacêutica, orientações maneira necessárias (Febrafar, 2019).

A Federação Internacional de Farmacêuticos (FIF), descreve que a farmácia comunitária é o primeiro local frequentado por pessoas acometidas por uma enfermidade, e necessidades de atenção e cuidados. Logo, os países afetados ou não por uma pandemia devem considerar as farmácias comunitárias como parceiras durante os esforços para que não ocorra uma contaminação em massa, considera-se o serviço farmacêutico como uma prestação de serviços na orientação e cuidadosa saúde (FIF, 2017)

De maneira evolutiva quando a farmácia comunitária oferece um serviço, além de atender uma demanda do paciente, contribui para diminuir o número de pessoas nos postos de saúde e hospitais, medida essencial durante uma pandemia que é evitar as aglomeração de pessoas (Amariles et al., 2020). 
A Agência Nacional de Vigilância Sanitária (ANVISA), iniciou no mês de agosto de 2019 revisões na RDC 44 com o objetivo de ampliar os serviços ofertado sem farmácias e drogarias. Aonde farmacêuticos aguardam com bastante expectativa a aprovação de novos serviços e evoluções em que o órgão classifique como serviços todas as suas atribuições dentro da empresa (ANVISA, 2019).

\section{A importância da farmácia clínica e o cuidado farmacêutico}

A farmácia clínica surgiu nos anos 60 nos Estados Unidos em ambiente hospitalar, os resultados positivos fizeram romper barreiras e chegar ao recinto da maioria das farmácias na Europa, Estados Unidos e América do Sul. No Brasil a Lei 13.021/2014, altera o papel das farmácias, antes definidas como responsáveis por comercializar medicamentos e correlatos, agora passam ser unidades de prestaçãodeserviçosfarmacêuticos,dispensandomedicamentos,orientandoeacompanhando os pacientes durante o tratamento,sendo parte fundamental na manutenção de uma saúde com qualidade (Brasil, 2017).

$\mathrm{O}$ avanço das atividades clínicas do farmacêutico é uma resposta às mudanças demográficas e epidemiológicas observadas na sociedade. O farmacêutico da atualidade trabalha como cuidado direto ao paciente, promovendo o uso racional de medicamentos e de outras tecnologias em saúde, restabelecendo sua prática mediante as necessidades dos pacientes, família, cuidadores e sociedade (Nicoletti \& Ito, 2017; Saraiva et al., 2018).

O uso racional de medicamentos tornou-se uma preocupação constante dos órgãos de saúde. Logo o conhecimento e matenção farmacêutica coloca o farmacêutico em evidência para realizar atendimento e orientação a população para o uso correto de medicamentos. Essa iniciativa dentro das farmácias proporciona fidelização de clientes, ocasionando um retorno financeiro satisfatório. Sendo a farmácia um estabelecimento de saúde, é imprescindível a presença de um profissional com conhecimento científico para coordenar as atividades, já que a maioria dos atendentes possuem apenas o conhecimento prático, insuficiente para um atendimento ideal e seguro (Oliveira et al.,2017).

\section{Educação em saúde na farmácia comunitária}

A educação é uma ferramenta de orientação no âmbito medicamentoso e no controle de doenças contribuindo na melhoria da qualidade de vida e saúde, exigindo o comprometimento de seus integrantes na condução desse processo, sendo assim precisa oferecer o acompanhamento correto ao paciente para que ele consiga conviver com a doença, e tenha uma melhor qualidade de vida (Brasil, 2017).

A pratica farmacêutica estabelece-se de maneira orientativa por via educacional em referência ao tratamento medicamentoso, essas orientações são necessárias para a criação de metodologias, a fim de melhorar os serviços prestados na farmácia comunitária, estabelecendo atividades assistências com o auxílio do cuidado farmacêutico, cooperando com a farmacoterapia individual do paciente (Melo et al.,2017).

\section{Serviços farmacêuticos realizados na farmácia comunitária privada}

Os serviços farmacêuticos, visa o cuidado ao paciente gerenciando da melhor maneira o uso de medicamentos voltados a atividades relacionadas a orientação, prestação de serviços e promoção de saúde (Correr,2019).

Além dos serviços, o profissional farmacêutico poderá prescrever os Medicamentos Isentos de Prescrição (MIPs), visando amenizar as dificuldades que as pessoas enfrentam para obter atendimento médico no Brasil, sendo esses medicamentos em sua maioria fitoterápicos, oferecendo tranquilidade a quem prescreve e principalmente aos pacientes que utilizam o medicamento (Brasil, 2019).

Os serviços farmacêuticos prestados nas farmácias comunitárias, possibilita a melhoria na qualidade de vida dos pacientes, além de apresentar ao público o profissional de saúde que é o farmacêutico. Como atendimento aumenta a 
fidelização de clientes e novas indicações acontecem devido a propaganda realizada por pacientes e profissionais que compartilham dos resultados positivos (ICTQ, 2019).

A população necessita de serviços farmacêuticos conforme a necessidade, dessa forma, o farmacêutico na farmácia privada busca melhorar a adesão ao tratamento com medicamentos realizando intervenções que esclareçam as informações sobre os tratamentos não medicamentosos como a mudança comportamental e alimentar (Silva et al.,2018).

Essas intervenções são executadas com o objetivo de precaver os erros de prescrição e de administração assim sendo,o farmacêutico clínico tem um papel essencial na promoção do uso racional do medicamento, por meio da garantia da farmacoterapia apropriada, com resultados terapêuticos seguros e minimizando os resultados contrários (Nicoletti \& Ito, 2017).

As intervenções devem ser planejadas, documentadas e executadas junto ao paciente e profissionais de saúde, que tem como visão principal sanar ou prevenir problemas que interferem ou podem interferir na farmacoterapia, constituindo-se parte complementar do procedimento de acompanhamento/seguimento farmacoterapêutico (Nicoletti \& Ito, 2017).

\section{Metodologia}

Trata-se de uma revisão integrativa fundamentada na pesquisa bibliográfica, método que agrupa a produção científica relevante acerca de um tema pré estabelecido (Estrela, 2018), ofertando acesso rápido e sintetizado aos resultados científicos de maior acuidade para a área estudada, estabelecida por meio de artigos publicados sobre a importância do farmacêutico clínico na farmácia comunitária privada.A coleta de dados foi realizada no período de fevereiro e março de 2021, onde formão investigados estudos publicados nas bases Cochrane Library, Epistemonikos, Biblioteca Virtual de Saúde e Google Scholar, utilizando-se os descritores ("clinical pharmacist and private community pharmacy") e ("pharmaceutical services and health education”), adaptados ao Medical Subject Headings (MeSH) e Descritores em Ciências da Saúde (DeCS), associados com operadores boleanos "AND" e "OR". A pesquisa foi limitada as línguas portuguesa, espanhola e inglesa, adotando-se um recorte temporal de 2015 a 2021.

Dos artigos selecionados foram retiradas as seguintes informações a fim de sumarizar os resultados encontrados: autores, ano de publicação, local onde foi realizado o estudo, desenho do estudo e resultados alcançados.

No tocante aos critérios de inclusão foram incluídos os artigos publicados nos últimos 5 anos que abordem a importância do farmacêutico na farmácia comunitária privada e estão disponibilizados eletronicamente na íntegra e foram excluídos os artigos que foram publicados fora do limite temporal 2015- 2021, que tratam da importância do farmacêutico e mostram áreas de atuação que não seja farmácia comunitária privada, e que não estejam disponíveis eletronicamente.

\section{Resultados e Discussão}

14 artigos foram selecionados, sendo 08 deles revisões de literatura, 01 estudo transversal, 01 revisão sistemática com síntese narrativa e 04 estudos bibliográficos integrativos, sendo um dele com estudo quantitativo. Os estudos contemplem e sintetizam a importância do farmacêutico clínico na farmácia comunitária privada.

A Tabela 1, seguinte, contempla os artigos selecionados e descreve suas características. 
Tabela 1 - Perfil e características dos artigos selecionados (Continua).

\begin{tabular}{|c|c|c|c|}
\hline TÍTULO & OBJETIVO & $\begin{array}{c}\text { METODOLOGIA } \\
\text { DO ESTUDO }\end{array}$ & RESULTADOS \\
\hline $\begin{array}{l}\text { O farmacêutico clínico na } \\
\text { farmácia comunitária }\end{array}$ & $\begin{array}{llr}\text { Descrever o papel do } \\
\text { farmacêutico } \\
\text { farmácínico comunitária }\end{array}$ & Revisão bibliográfica & $\begin{array}{l}\text { Compreendeu-se que a farmácia comunitária não deve } \\
\text { ser tratada apenas como estabelecimento comercial, mas } \\
\text { sim como um local de promoção a saúde, onde a atuação } \\
\text { do farmacêutico contribui para a diminuição na } \\
\text { sobrecarga do sistema de saúde. Para a ótima atuação do } \\
\text { farmacêutico em farmácia comunitária é essencial que o } \\
\text { profissional esteja sempre atualizado sobre as legislações } \\
\text { vigentes para o cumprimento de suas funções. }\end{array}$ \\
\hline $\begin{array}{l}\text { Farmácia clínica em } \\
\text { ambiente }\end{array}$ & $\begin{array}{l}\text { Verificar a percepção e a } \\
\text { prática do farmacêutico sobre o } \\
\text { registro das atividades clínicas. }\end{array}$ & Estudo transversal & $\begin{array}{l}\text { Dentre os } 27 \text { profissionais que compunham a amostra, foi } \\
\text { possível contatar } 26.73 \% \text { dos farmacêuticos convidados } \\
\text { participaram do estudo. Observou-se um perfil de } \\
\text { farmacêuticos jovens }(24 \text { a } 35 \text { anos), em sua maior parte } \\
\text { do sexo feminino }(94,74 \%) \text {, com formação recente }(01 \\
\text { mês a } 12 \text { anos) e em instituição privada }(83,16 \%) \text {. } \\
\text { Grande parte destes farmacêuticos }(57,89 \% \text { dos } \\
\text { farmacêuticos) atua em mais de um setor (farmácias } \\
\text { internas, satélites e central) }\end{array}$ \\
\hline $\begin{array}{l}\text { Importância da farmácia } \\
\text { clínica para a identificação } \\
\text { e resolução de problemas } \\
\text { relacionados } \\
\text { medicamentos (PRM) a }\end{array}$ & $\begin{array}{l}\text { Realizar uma revisão literária } \\
\text { sobre a importância da farmácia } \\
\text { clínica para a identificação e } \\
\text { solução de } \text { Problemas } \\
\text { Relacionados a Medicamentos } \\
\text { (PRM). }\end{array}$ & Revisão de literatura & $\begin{array}{l}\text { Constatou-se que os Problemas Relacionados a } \\
\text { Medicamentos (PRM) são a principal causa de eventos } \\
\text { adversos no ambiente hospitalar que podem ser } \\
\text { prevenidos. As Intervenções Farmacêuticas (IF) } \\
\text { realizadas mostraram-se importantes para a redução dos } \\
\text { PRMs, minimizando os resultados terapêticos } \\
\text { desfavoráveis, melhorando a qualidade dos cuidados com } \\
\text { o paciente, aumentando a segurança da farmacoterapia, e, } \\
\text { ao mesmo tempo, reduzindo os custos e o tempo da } \\
\text { internação. }\end{array}$ \\
\hline $\begin{array}{l}\text { Orientação farmacêutica na } \\
\text { dispensação de } \\
\text { antimicrobianos em uma } \\
\text { farmácia comunitária de } \\
\text { encruzilhada do SUL/RS }\end{array}$ & $\begin{array}{l}\text { Avaliar a } \text { orientação } \\
\text { farmacêutica na dispensação de } \\
\text { antimicrobianos em uma } \\
\text { farmácia comunitária do } \\
\text { município de } \\
\text { Sul. Encruzilhada do }\end{array}$ & $\begin{array}{l}\text { Revisão bibliográfica } \\
\text { e estudo quantitativo }\end{array}$ & $\begin{array}{l}\text { Os resultados mostraram que a média geral da satisfação } \\
\text { com a orientação farmacêutica foi de } 67,5( \pm 3,5) \text {. A } \\
\text { média geral de respostas "excelente" foi de } 53 \% \text {. A } \\
\text { maioria dos entrevistados foram do sexo feminino }(58 \%) \\
\text { e tinham em média } 38,12( \pm 13) \text { anos. Concluindo que é } \\
\text { fundamental a atuação correta do profissional } \\
\text { farmacêutico para conscientizar os usuários sobre o uso } \\
\text { racional de medicamentos, principalmente os } \\
\text { antimicrobianos. }\end{array}$ \\
\hline $\begin{array}{l}\text { Cuidados farmacêuticos na } \\
\text { farmácia comunitária }\end{array}$ & $\begin{array}{l}\text { Definir os cuidados } \\
\text { farmacêuticos, observar a sua } \\
\text { evolução ao longo das ultimas } \\
\text { décadas e constatar os } \\
\text { benefícios desta pratica. }\end{array}$ & Revisão de literatura & $\begin{array}{l}\text { Constatou-se que } \mathrm{O} \text { cuidado farmacêutico é o } \\
\text { fornecimento responsável de terapia medicamentosa com } \\
\text { o objetivo de alcançar resultados definidos que melhorem } \\
\text { a qualidade de vida do doente. Envolve o processo pelo } \\
\text { qual o farmacêutico colabora com o doente e outros } \\
\text { profissionais na concepção, implementação e } \\
\text { monitorização de um plano terapêutico que ira produzir } \\
\text { um resultado terapêutico especifica para o paciente. }\end{array}$ \\
\hline $\begin{array}{l}\text { Cuidado farmacêutico para } \\
\text { utentes de farmácia } \\
\text { comunitária privada: } \\
\text { Revisão sistemática }\end{array}$ & $\begin{array}{l}\text { Sintetizar evidências sobre } \\
\text { intervenções farmacêuticas } \\
\text { que proporciona aos utentes } \\
\text { de farmácia comunitária } \\
\text { privada tratamentos eficientes e } \\
\text { com a melhoria da qualidade de } \\
\text { vida. }\end{array}$ & $\begin{array}{c}\text { Revisão } \\
\text { Sistemática e } \\
\text { síntese narrativa. }\end{array}$ & $\begin{array}{l}\text { Observou-se redução da prescrição de medicamentos } \\
\text { potencialmente inapropriados, melhoria do uso de } \\
\text { medicamentos apropriados e seguros, melhoria da adesão } \\
\text { ao tratamento, redução de eventos adversos a } \\
\text { medicamentos, de interações medicamentos- } \\
\text { medicamentos e de resultados negativos em saúde } \\
\text { associados a medicamentos, redução de consultas } \\
\text { ambulatoriais, de visitas a serviços de urgências e } \\
\text { emergências, de internações hospitalares, de } \\
\text { morbidade, mortalidade, melhoria do estado de saúde, da } \\
\text { qualidade de vida e redução de custos de medicamentos. }\end{array}$ \\
\hline $\begin{array}{l}\text { Seguimento } \\
\text { farmacoterapêutico nas } \\
\text { farmácias comunitárias } \\
\text { privadas: instrumento para } \\
\text { fortalecimento da prática } \\
\text { clínica e efetividade do } \\
\text { tratamento do paciente }\end{array}$ & $\begin{array}{l}\text { Esclarecer os benefícios do uso } \\
\text { do Seguimento } \\
\text { Farmacoterapêutico nas } \\
\text { farmácias comunitárias } \\
\text { privadas como instrumento para } \\
\text { o fortalecimento da prática } \\
\text { clínica e efetividade do } \\
\text { tratamento do paciente }\end{array}$ & Revisão bibliográfica & $\begin{array}{l}\text { A farmácia comunitária é um espaço propício à prática } \\
\text { clínica do farmacêutico que, por meio do Seguimento } \\
\text { Farmacoterapêutico (SFT) e raciocínio clínico do } \\
\text { fármaco, identifica patologias não tratadas, doses } \\
\text { subterapêuticas, interações, acompanhando, com } \\
\text { propostas de medidas de redução e efetividade, na adesão } \\
\text { de pacientes com HAS e DMT2. Apesar das dificuldades, } \\
\text { a Atenção Farmacêutica nas farmácias comunitárias pelo } \\
\text { uso do Seguimento Farcoterapêutico é um meio que } \\
\text { fortalece a prática clínica, contribuindo para a obtenção } \\
\text { de resultados efetivos da farmacoterapia }\end{array}$ \\
\hline
\end{tabular}




\begin{tabular}{|c|c|c|c|}
\hline $\begin{array}{l}\text { Polimedicação, atenção } \\
\text { farmacêutica e Cuidado } \\
\text { farmacêutico }\end{array}$ & $\begin{array}{l}\text { Discutir a importância do } \\
\text { farmacêutico para minimizar os } \\
\text { impactos causados pela } \\
\text { polifarmácia e esclarecer sobre } \\
\text { o uso racional de fármacos, } \\
\text { assim com o discorrer sobre } \\
\text { a utilização dos termos } \\
\text { atenção farmacêutica e } \\
\text { cuidado farmacêutico. }\end{array}$ & Revisão de literatura & $\begin{array}{l}\text { A polimedicação pode ocasionar inúmeros riscos à saúde, } \\
\text { uma vez que pode aumentar a toxicidade do } \\
\text { medicamento, anular o seu efeito ou até mesmo } \\
\text { potencializar a ação de outro fármaco utilizado } \\
\text { concomitantemente. Dessa forma, a atuação do } \\
\text { farmacêutico é de suma importância no acompanhamento } \\
\text { do usuário da polifarmácia, tendo em vista que sua } \\
\text { atuação proporciona uma evidente melhora na } \\
\text { qualidade de vida do paciente. }\end{array}$ \\
\hline $\begin{array}{llr}\text { Contribuição } & \text { do } \\
\text { farmacêutico para } & \text { a } \\
\text { promoção de saúde na } \\
\text { farmácia comunitária }\end{array}$ & $\begin{array}{l}\text { Descrever as contribuições do } \\
\text { farmacêutico para a promoção } \\
\text { de saúde na farmácia } \\
\text { comunitária }\end{array}$ & Revisão de literatura & $\begin{array}{l}\text { O uso irracional de medicamentos é um importante } \\
\text { problema de saúde pública; portanto, é preciso considerar } \\
\text { o potencial de contribuição do farmacêutico e } \\
\text { efetivamente incorporá-lo às equipes de saúde a fim de } \\
\text { que se garanta a melhoria da utilização dos } \\
\text { medicamentos, com redução dos riscos de mortalidade e } \\
\text { que seu trabalho proporcione meios para que os custos } \\
\text { relacionados à farmacoterapia sejam os menores } \\
\text { possíveis para a sociedade. }\end{array}$ \\
\hline $\begin{array}{l}\text { Relação farmacêutico- } \\
\text { paciente a partir do olhar } \\
\text { clínico }\end{array}$ & $\begin{array}{l}\text { Apresentar a importância da } \\
\text { relação farmacêutico-paciente, } \\
\text { com um olhar clínico } \\
\text { fundamentado, ressaltando a } \\
\text { dimensão da Atenção } \\
\text { Farmacêutica (AF) na } \\
\text { orientação para uso racional de } \\
\text { medicamentos. }\end{array}$ & Revisão bibliog & $\begin{array}{l}\text { Através de uma abordagem bibliográfica, buscou-se } \\
\text { reunir informações pertinentes a atuação farmacêutica } \\
\text { nas suas atribuições legais, visando ressaltar a relevância } \\
\text { do atendimento e orientação ao paciente de forma } \\
\text { responsável e baseada em evidências, para que a } \\
\text { profissão farmacêutica seja cada vez mais valorizada e } \\
\text { tenha reconhecimento social como profissional da saúde } \\
\text { que pode oferecer um serviço capaz de promover a saúde } \\
\text { do paciente de forma segura e eficaz, contribuindo } \\
\text { também para redução de visitas desnecessárias a } \\
\text { unidades de saúde, e, consequentemente diminuindo a } \\
\text { superlotação das mesmas, que atualmente pode ser } \\
\text { considerada um problema de saúde pública. }\end{array}$ \\
\hline $\begin{array}{l}\text { Implantação de } \text { serviços } \\
\text { farmacêuticos } \\
\text { farmácias e drogarias: uma } \\
\text { revisão de literatura }\end{array}$ & 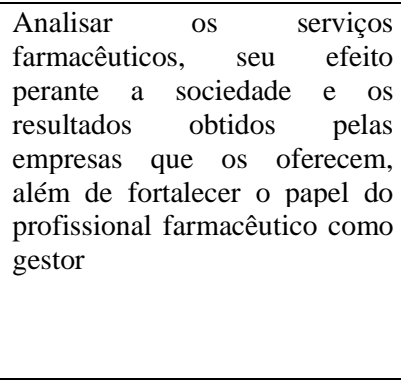 & Revis & $\begin{array}{l}\text { Através do estudo realizado em artigos e publicações } \\
\text { sobre o tema, constatou-se que os serviços farmacêuticos } \\
\text { ainda é uma realidade distante para a maioria dos } \\
\text { pacientes e farmacêuticos. Os serviços prestados nas } \\
\text { farmácias e drogarias além de trazer qualidade de vida } \\
\text { para os pacientes, fortalece junto à comunidade o } \\
\text { profissional farmacêutico. Além disso, percebe-se que a } \\
\text { implementação dos serviços em farmácias comunitárias } \\
\text { esbarra em dificuldades financeiras e falta de tempo dos } \\
\text { farmacêuticos que tem que se dividir entre o atendimento } \\
\text { no balcão e a consulta. }\end{array}$ \\
\hline $\begin{array}{ll}\text { Farmácia } & \text { clínica: } \\
\text { importância deste } & \text { serviço } \\
\text { no cuidado a saúde } & \end{array}$ & $\begin{array}{l}\text { Verificar a importância do } \\
\text { serviço de farmácia clínica no } \\
\text { cuidado à saúde. }\end{array}$ & Revisão de literatura & $\begin{array}{l}\text { As intervenções realizadas pelo farmacêutico clínico são } \\
\text { capazes de promover melhores resultados terapêuticos, } \\
\text { garantindo segurança, eficácia e custo-efetividade da } \\
\text { farmacoterapia. Este profissional na atenção farmacêutica } \\
\text { proporciona o uso racional de medicamentos, além de } \\
\text { interagir com outros profissionais de saúde, para um } \\
\text { adequado acompanhamento ao paciente no cuidado à } \\
\text { saúde. As evidências demonstradas neste estudo } \\
\text { reforçam a importância do serviço de farmácia clínica no } \\
\text { acompanhamento e orientação do farmacêutico aos } \\
\text { pacientes com problemas relacionados a medicamentos. }\end{array}$ \\
\hline \begin{tabular}{lrr} 
Serviços & \multicolumn{2}{c}{ farmacêuticos } \\
clínicos & na & atenção \\
primária à saúde do Brasil
\end{tabular} & $\begin{array}{llr}\text { Analisar os } & \text { tipos } & \text { e os } \\
\text { benefícios } & \text { dos } & \text { serviços } \\
\text { farmacêuticos } & & \text { clínicos } \\
\text { desenvolvidos } & \text { na } & \text { atenção } \\
\text { primária à saúde do Brasil }\end{array}$ & Revisão de literatura & $\begin{array}{l}\text { No constante da esteira dos benefícios, a literatura } \\
\text { demonstra a coexistência, a importância e a } \\
\text { multidimensionalidade dos serviços farmacêuticos } \\
\text { clínicos na promoção da saúde e do uso racional de } \\
\text { medicamentos pela comunidade adstrita. }\end{array}$ \\
\hline $\begin{array}{l}\text { Serviços farmacêuticos nos } \\
\text { países incluídos no Health } \\
\text { In Transition(HIT): uma } \\
\text { análise comparada }\end{array}$ & $\begin{array}{l}\text { Analisar a conformação dos } \\
\text { serviços farmacêuticos em } \\
\text { quatro países da União } \\
\text { Européia -Espanha, França, } \\
\text { Portugal e Itália. }\end{array}$ & Revisão de literatura & $\begin{array}{l}\text { Os dados indicam que as políticas de saúdetêm muitas } \\
\text { similaridades quanto à regulamentação, enquanto } \\
\text { sepercebemdiferenças quantoàs políticas de copagamento } \\
\text { e fixação de preços, que, consequentemente, interferem } \\
\text { na estruturação dos serviços farmacêuticos em seus } \\
\text { sistemas de saúde. }\end{array}$ \\
\hline
\end{tabular}

Fonte: Autores (2021).

A partir dos estudos selecionados iniciando no contexto dos artigos que tratam da Importância do Farmacêutico na farmácia comunitária, Sarmento et al (2011) constatou por meio de uma revisão de literatura que o profissional farmacêutico é 
de fundamental importância para a vida dos pacientes, sendo o responsável pelo manejo, dispensação e orientação medicamentosa.

De Lima et al (2017) demonstraram através de um estudo transversal que a maior parte dos profissionais farmacêuticos já atuantes, trabalham em áreas variadas, tais como farmácias internas, satélites e central. No artigo Importância da farmácia clínica para a identificação e resolução de problemas relacionados a medicamentos (PRM), Albuquerque Junior et al (2021) demonstram que os problemas relacionados a medicamentos podem ser resolvidos através da Intervenção farmacêutica, evidenciando a importância deste profissional através da minimização das falhas através da sua atuação.

Custodio (2019) evidencia mais uma vez essa atuação do farmacêutico, e a importância do seu papel na orientação correta sobre o uso de medicamentos. Costa (2020) demonstrou que o cuidado farmacêutico melhora a qualidade de vida dos pacientes, sendo o orientador no uso dos medicamentos.

Da Cruz, Queiroz e Soler (2020) demonstrou em seu estudo a melhora no uso de medicamentos, e diminui os usos negativos de medicamentos, além de melhorar a saúde e a qualidade de vida dos usuários. Lima et al (2019) demostram que o seguimento farmacoterapêutico é um meio de fortalecer a pratica e atuação do farmacêutico na farmácia clinica comunitária, contribuindo para os resultados efetivos.

Batista et al (2020) trata da polimedicação, e da importância da orientação farmacêutica, demonstrando os riscos que este ato traz para a saúde dos pacientes, potencializando a atuação do farmacêutico na orientação da utilização dos fármacos. Lopes (2020) também pontuam a importância do farmacêutico na orientação medicamentosa, demonstrando que o uso exacerbado de medicamentos pode trazer danos a saúde, viabilizando a incorporação do farmacêutico em equipes de saúde.

De Sousa e Trevisan (2021) apresentam a importância da relação entre farmacêutico e paciente, demonstrando que a atuação do farmacêutico melhora significativamente os tratamentos, viabiliza o uso de medicamentos, e diminui as idas dos pacientes até as unidades de saúde. Da Silva et al (2017) realizaram uma pesquisa acerca da existência e instalação de farmácias no Brasil, foi possível identificar que os serviços farmacêuticos ainda estão longe de todos os pacientes, ou seja, nem todos tem acesso a este serviço, isso decorre do fato de a instalação e implementação de farmácias enfrenta grandes barreiras.

Barros et al (2019) demonstram que as intervenções realizadas pelo farmacêutico clínico são capazes de promover melhores resultados terapêuticos, garantindo segurança, eficácia e custo-efetividade da farmacoterapia. Sendo o profissional farmacêutico o responsável pelo acompanhamento adequado ao paciente.

De Lima et al (2017) demonstram que a literatura em seu constante demonstra e viabiliza a importância dos serviços farmacêuticos clínicos na promoção da saúde e do uso racional de medicamentos pela comunidade. Sousa et al (2020) tratam dos serviços de saúde em países da União Europeia, demonstrando que as políticas de saúde têm muitas similaridades quanto à regulamentação, enquanto se percebem diferenças quanto às políticas de copagamento e fixação de preços, que, consequentemente, interferem na estruturação dos serviços farmacêuticos em seus sistemas de saúde.

\section{Conclusão}

As evidências apresentadas neste estudo demonstram a ação do farmacêutico clinico na farmácia comunitária, demonstrando que cabe a este profissional o manejo, dispensação e orientação quanto ao uso de medicamentos. Em todos os artigos aqui apresentados nesta revisão integrativa demonstram a importância deste profissional no que condiz a saúde, a segurança, o bem estar e a qualidade de vida dos pacientes.

Dessa forma, como sugestão para trabalhos futuros colocamos a intenção de analisar a percepção dos farmacêuticos clínicos acerca dos serviços prestados em farmácias comunitárias privativas, analisando dessa forma as condições e os trabalhos que são prestados por estes profissionais. 


\section{Implicações práticas}

Recomenda-se a necessidade de estudos complementares quanto a atuação do farmacêutico clinico em farmácias comunitárias privadas, a fim de aumentar a literatura acerca dessa temática.

\section{Declaração de conflito de interesses}

$\mathrm{O}$ autor declara que essa pesquisa foi conduzida na ausência de quaisquer relações comerciais ou financeiras que possam ser interpretados como um potencial conflito de interesse.

\section{Referências}

Albuquerque, L. B. J, et al. (2013). Importancia da farmácia clinica para a identificação e resolução de problemas relacionados a medicamentos (PRM). Revista Saúde em Foco, 13 (13), 9 - 20. https://portal.unisepe.com.br/unifia/wp-content/uploads/sites/10001/2021/01/IMPORT\%C3\%82NCIA-DAFARM\%C3\%81CIA-CL\%C3\%8DNICA-PARA-A-IDENTIFICA\%C3\%87\%C3\%83O-E-RESOLU\%C3\%87\%C3\%83O-DE-PROBLEMASRELACIONADOS-A-MEDICAMENTOS-PRM-9-\%C3\%A0-20.pdf.

Amariles, P., Ledezma-Morales, M., Salazar-Ospina, A., \& Hincapié-García, J. A. (2021). Como vincular pacientes com suspeita de COVID-19 ao sistema de saúde das farmácias comunitárias? Uma proposta de rota. Research in Social and Administrative Pharmacy, 17 (1), 19881989.http://dx.doi.org/10.1016/j.sapharm.2020.03.007.

Barros, D.S. \& Silva, D. L. M. (2020). Serviços farmacêuticos clínicos na atenção primária à saúde do Brasil. Trab. educ. saúde, 18 (1). https://doi.org/10.1590/1981-7746-sol00240 .

Batista, S. C., Albuquerque, L. E., Silva, N. M., Santos, B. G. C., Medeiros, J. S. (2020) Polimedicação, atenção farmacêutica e Cuidado farmacêutico. Journal of Biology \& Pharmacy and Agricultural Management, 16 (4), 455 - 469. http://revista.uepb.edu.br/index.php/biofarm/article/view/5657/3657.

Brasil. (2019). Presidência da República. Secretaria-geral Subchefia Para Assuntos Jurídicos (Org.). Lei nº 13.874, de 20 de setembro de 2019. Declaração de direitos de liberdade econômica, Brasília-DF, v.1,n.1,p.1-1.

Britto Sousa I, Nunes Carvalho V, Pereira Campos dos Santos HL, de Brito Lima Prado NM. (2021). Serviços farmacêuticos nos países incluídos no Health In Transition (HIT): uma análise comparada. Rev. G \& amp;11 (3), 310-25. https://periodicos.unb.br/index.php/rgs/article/view/32180

Correia, K. K. L., Barros, M. L. C. M., Barros, M. R., Marques, R. A. (2017). Farmácia clínica: importância deste serviço no cuidado a saúde. Boletim Informativo Geum, 8 (3), 7 - 18. https://revistas.ufpi.br/index.php/geum/article/view/6183/4256.

Custódio, S. S. (2019). Orientação farmacêutica na dispensação de antimicrobianos em uma farmácia comunitária de Encruzilhada do Sul - RS. In: Trabalho de Conclusão de Curso, Curso de Farmácia, Universidade de Santa Cruz do Sul. https://repositorio.unisc.br/jspui/handle/11624/2658.

Cruz, Wilcléa Mendes da., Queiroz, Luana Melo Diogo de., Sole, Orenzio. (2020) Cuidado farmacêutico para utentes de farmácia comunitária privada: revisão sistemática. Brazilian Journal of Development, 6 (10), 78682 - 78702. https://www.brazilianjournals.com/index.php/BRJD/article/view/18343/14803.

Febrafar (2019). Mercado farmacêutio 2019 - veja expectativas. Projeção para 2019, São Paulo-SP. 1(1), 1-1. https://www.febrafar.com.br/mercadofarmaceutico-2019-veja-expectativas.

Ferreira, M. S. (2020). Implantação de serviços farmacêuticos em farmácias e drogarias: uma revisão de literatura. In: FAMAM - Trabalhos de Conclusão de Curso, Curso de Farmácia. http://famamportal.com.br:8082/jspui/handle/123456789/1884.

ICTQ (2019). Seviços farmacêuticos - A nova aposta na competição do varejo. Suporte Diferenciado, Anápolis, p.1-1. https://www.ictq.com.br/varejofarmaceutico/775-servicos-farmaceuticos-a-novaaposta-na-competicao-do-varejo.

Lima, E. D, et AL. (2017) Farmácia clinica em ambiente hospitalar: enfoque no registro das atividades. Rev. Bras. Farm. Hosp. Serv. Saúde, 8 (4), 18 - 24. http://www.v1.sbrafh.org.br/public/artigos/2017080403001253ES.pdf.

Lima, J. P, et AL. (2019). Seguimento farmacoterapêutico nas farmácias comunitárias privadas: instrumento para fortalecimento da prática clínica e efetividade do tratamento do paciente. In: Encontro de extensão, docência e iniciação científica, 2 - 6 .

Nicoletti, M. A. \& Ito, K. R. (2017). Formação do farmacêutico: Novo cenário de atuaçãoprofissional com o empoderamento de atribuições clínicas. Revista Saúde. 11(3), 49-62. http://revistas.ung.br/index.php/saude/article/view/2536.

Oliveira, N.; Villas Boas, V. et AL (2017). Atuação profissional dos farmacêuticos no Brasil: perfil sócio demográfico e dinâmica de trabalho em farmácias e drogarias privadas. Revista Saúde e Sociedade. 26, (4), 1105-1121. http://dx.doi.org/10.1590/s0104-12902017000002 Acesso em:30de março de2021.

Rafael, E. S, et AL. (2016) Contribuição do farmacêutico para a promoção de saúde na farmácia comunitária. In: XI Mostra Científica da Farmácia, 3 (2), 2 4. http://publicacoesacademicas.unicatolicaquixada.edu.br/index.php/mostracientificafarmacia/article/view/1797/1513.

Sarmento, D. P, et AL. (2020). O farmacêutico clinico na farmácia comunitária. Revista Eletrônica Gestão e Saúde, 1 (1), 60-75. https://administradoresdevalor.com.br/images/revista/4-o-farmaceutico-clinico-na-farmacia-comunitaria.pdf.

Saraiva,e.m.s.;Alencar, g.o.;Machado,y.s.o. \& Gomes,a.e.B. (2018) Uso da Estratégia Aprendizagem Baseada em Equipes em Disciplinas da Graduação em Farmácia. Revista Multiprofissional Psicologia. 12 (39), 831-838. 
Research, Society and Development, v. 10, n. 13, e558101321515, 2021

(CC BY 4.0) | ISSN 2525-3409 | DOI: http://dx.doi.org/10.33448/rsd-v10i13.21515

Silva,E.Letal (2018). Prevalência da Adesão Terapêutica em Pacientes Hipertensos sob Tratamento Medicamentoso em um Município do Sudoeste Baiano. Id On Line Rev.Mult.Psic,12. (42), 749-759.

Sousa, F. V. \& Trevisan, M. (2021). Relação farmacêutico-paciente a partir do olhar clínico. Revista Artigos. Com, 29, e7632. https://acervomais.com.br/index.php/artigos/article/view/7632 